Bull. Austral. Math. Soc.

VoL. 42 (1990) [153-155]

\title{
MULTIPLICATTVITY OF THE UNIFORM NORM AND INDEPENDENT FUNCTIONS
}

\section{A. GuYan Robertson}

It has long been known that there is a close connection between stochastic independence of continuous functions on an interval and space-filling curves [9]. In fact any two nonconstant continuous functions on $[0,1]$ which are independent relative to Lebesgue measure are the coordinate functions of a space filling curve. (The results of Steinhaus [9] have apparently been overlooked in more recent work in this area [3, 5, 6].)

The purpose of this note is to establish a connection with a multiplicative property for the uniform norm on function spaces. Only the uniform norm will be considered on $C(\Omega)$, the space of continuous real valued functions on a compact Hausdorf space $\Omega$. If $f \in C(\Omega)$ then we define $Q(f)$ to be the linear span of $\left\{1, f, f^{2}\right\}$, that is the space of quadratic polynomials in $f . \Omega(f)$ is known to play a role in the isometric theory of $C(\Omega)$. For example, if $f$ separates points of $\Omega$ then $Q(f)$ is a Korovkin set; so if $\varphi$ is a contraction on $C(\Omega)$ which fixes $Q(f)$ then $\varphi$ is the identity map $[1,7,8]$.

PROPOSITION 1. Let $\Omega$ be a compact Hausdorff space, let $f_{j} \in C(\Omega)(j=1,2)$ and define $\gamma: \Omega \rightarrow \mathbf{R}^{2}$ by $\gamma(t)=\left(f_{1}(t), f_{2}(t)\right)$. The following statements are equivalent:

(a) $\gamma(\Omega)=f_{1}(\Omega) \times f_{2}(\Omega)$,

(b) $\left\|g_{1} g_{2}\right\|=\left\|g_{1}\right\|\left\|g_{2}\right\|$ for all $g_{j} \in Q\left(f_{j}\right)(j=1,2)$.

Proof: (b) $\Rightarrow\left(\right.$ a). Let $\left(x_{1}, x_{2}\right) \in f_{1}(\Omega) \times f_{2}(\Omega)$. There exist quadratic polynomials $p_{j}$ such that $p_{j}\left(x_{j}\right)=1$ and $\left|p_{j}(x)\right|<1$ for $x \in f_{j}(\Omega) \backslash\left\{x_{j}\right\}$. Let $g_{j}=p_{j} \circ f_{j}$, so that $g_{j} \in Q\left(f_{j}\right)(j=1,2)$.

By hypothesis, $1=\left\|g_{1}\right\|\left\|g_{2}\right\|=\left\|g_{1} g_{2}\right\|$. It follows that there is a point $t \in \Omega$ such that $\left|g_{1}(t) g_{2}(t)\right|=1$, whence $f_{1}(t)=x_{1}$ and $f_{2}(t)=x_{2}$. This proves (a).

(a) $\Rightarrow(b)$. Any quadratic polynomial $p_{j}$ attains its maximum absolute value on $f_{j}(\Omega)$ at some point $x_{j}$. Our assumption implies that there is a point $t \in \Omega$ with $f_{j}(t)=x_{j}(j=1,2)$. Writing $g_{j}=p_{j} \circ f_{j}$ we see that $\left|g_{1}(t) g_{2}(t)\right|$ takes the value $\left\|g_{1}\right\|\left\|g_{2}\right\|$, and the result follows.

Received 19 October 1989

Copyright Clearance Centre, Inc. Serial-fee code: 0004-9729/90 \$A2.00+0.00. 
REMARK. Simple examples show that the condition $\left\|f_{1} f_{2}\right\|=\left\|f_{1}\right\|\left\|f_{2}\right\|$ is not sufficient to imply (a).

We now show how independence of functions gives rise to the multiplicative property of the norm. A Borel measure $\mu$ on $\Omega$ is said to be faithful if $\mu(G)>0$ for each nonempty open subset $G$ of $\Omega$. We refer to [2] for details on the notion of independence.

Proposition 2. Let $\Omega$ be a compact Hausdorff space and $\mu$ a faithful Borel probability measure on $\Omega$. Let $f_{1}$ and $f_{2}$ be functions on $\Omega$ that are $\mu$-independent and such that $\left|f_{1}\right|$ and $\left|f_{2}\right|$ are lower semicontinuous. Then $\left\|f_{1} f_{2}\right\|=\left\|f_{1}\right\|\left\|f_{2}\right\|$.

Proof: We may suppose that $f_{1}$ and $f_{2}$ are both nonzero.

Let $\varepsilon>0$ and let $V_{j}=\left\{t \in \Omega:\left|f_{j}(t)\right|>\left\|f_{j}\right\|-\varepsilon\right\}$. Then $\mu\left(V_{j}\right)>0$, since $V_{j}$ is a nonempty open set $(j=1,2)$. By independence of $\left|f_{1}\right|$ and $\left|f_{2}\right|$, we have

$$
\mu\left(V_{1} \cap V_{2}\right)=\mu\left(V_{1}\right) \mu\left(V_{2}\right)>0 .
$$

In particular $V_{1} \cap V_{2} \neq \emptyset$. It follows that there exists $t \in \Omega$ such that $\left|f_{j}(t)\right|>\left\|f_{j}\right\|-\varepsilon$ $(j=1,2)$. Therefore $\left\|f_{1} f_{2}\right\|>\left(\left\|f_{1}\right\|-\varepsilon\right)\left(\left\|f_{2}\right\|-\varepsilon\right)$. Since $\varepsilon>0$ was arbitrary this proves the result.

Now if we suppose that $f_{1}, f_{2} \in C[0,1]$ are independent relative to Lebesgue measure then the same is true for quadratic polynomials in $f_{1}$ and $f_{2}$, so Proposition 2 shows that condition (b) of Proposition 1 is satisfied. Thus if $f_{1}$ and $f_{2}$ are nonconstant then $\gamma[0,1]=f_{1}[0,1] \times f_{2}[0,1]$ is a rectangle in $\mathbf{R}^{2}$. (Note that the result of $[3]$ is a simple consequence of this fact.) Peano's original space-filling curve arises in this way $[9,6]$. In fact $[6]$ gives a detailed proof that the $n$-dimensional version of Peano's curve is measure preserving and hence has independent coordinate functions.

The step from independent coordinate functions to measure preserving mappings is often a small one.

Proposition 3. Let $f, g \in C[0,1]$ be independent and have continuous distribution functions $F, G$ respectively. Then the function $\varphi(t)=(F(f(t)), G(g(t)))$ defines a continuous measure preserving transformation of $[0,1]$ onto $[0,1] \times[0,1]$.

Proof: $F \circ f$ and $G \circ g$ are uniformly distributed over $[0,1]$, by $[2$, p.169 Exercise 14.4], and they are also independent since $f$ and $g$ are. The result follows easily.

In conclusion we recall that space filling curves arise naturally in functional analysis whenever we have a Hilbert space embedded isometrically in $C[0,1],[4,10]$. It is therefore interesting to note that the multiplicative norm condition fails in this case.

Proposition 4. Let $H \subset C[0,1]$ be a real Hilbert space, and let $f, g \in H$ be linearly independent. Then $\|f g\|<\|f\|\|g\|$.

Proof: The proof of [4] shows that if there is a point $t_{0} \in[0,1]$ such that $\left|f\left(t_{0}\right)\right|=$ $\|f\|$ and $\left|g\left(t_{0}\right)\right|=\|g\|$ then $f$ and $g$ must be linearly dependent. 


\section{REFERENCES}

[1] D. Amir and Z. Ziegler, 'Korovkin shadows and Korovkin systems in $C(S)$-spaces', $J$. Math. Anal. Appl. 62 (1978), 640-675.

[2] P. Billingsley, Probability and Measure (Wiley-Interscience, 1979).

[3] A.P. Bosznay, 'A remark on a paper of B.R. Gelbaum', Z. Wahrscheinlichkeitstheorie verw. Gebiete 43 (1978), 353-355.

[4] W.F. Donoghue Jr., 'Continuous function spaces isometric to Hilbert space', Proc. Amer. Math. Soc. 8 (1957), 1-2.

[5] B.R. Gelbaum, 'Independence of events and random variables', Z. Wahrscheinlichkeitstheorie verw. Gebiete 36 (1976), 333-343.

[6] S.C. Milne, 'Peano curves and smoothness of functions', Adv. in Math. 35 (1980), 129-157.

[7] W.M. Priestley, 'A noncommutative Korovkin theorem', J. Approx. Theory 16 (1976), 251-260.

[8] A.G. Robertson, 'A Korovkin theorem for Schwarz maps on $C^{*}$-algebras', Math. Z. 156 (1977), 205-207.

[9] H. Steinhaus, 'La courbe de Peano et les fonctions indépendantes', C.R. Acad. Sci. Paris 202 (1936), 1961-1963.

[10] D.T. Yost, 'Space filling curves and universal normed spaces', Ann. Univ. Sci. Budapest. Eötvös Sect. Math. 27 (1984), 39-42.

\footnotetext{
University of Edinburgh

Department of Mathematics

King's Buildings

Mayfield Road

Edinburgh EH9 3JZ

Scotland
} 